\title{
The Influence of Undergraduate's Mobile Phone Addiction on Learning Burnout: Based on Latent Moderated Structural Equation
}

\author{
Peng Ma, Bikai He, Wenhao Pan, Pengfei Qin, Shouying Zhao* \\ College of Psychology, Guizhou Normal University, Guiyang, China \\ Email: *zhaoshouying@126.com
}

How to cite this paper: Ma, P., He, B. K., Pan, W. H., Qin, P. F., \& Zhao, S. Y. (2020). The Influence of Undergraduate's Mobile Phone Addiction on Learning Burnout: Based on Latent Moderated Structural Equation. Psychology, 11, 966-979.

https://doi.org/10.4236/psych.2020.116062

Received: May 23, 2020

Accepted: June 27, 2020

Published: June 30, 2020

Copyright $\odot 2020$ by author(s) and Scientific Research Publishing Inc. This work is licensed under the Creative Commons Attribution International License (CC BY 4.0).

http://creativecommons.org/licenses/by/4.0/

\begin{abstract}
In order to explore the role of cognitive failure and anxiety between mobile phone addiction and learning burnout, 596 college students were surveyed with Anxiety scale, Cognitive Failure scale, Learning Burnout scale and Mobile Phone Addiction Tendency scale, which based on the latent moderated structural equation. The results show that: 1 ) The phone addiction has a positively significant effect on learning burnout; 2) The phone addiction can influence learning burnout through cognitive failure; 3) Anxiety can moderate the mediating role of cognitive failure.
\end{abstract}

\section{Keywords}

Learning Burnout, Phone Addiction, Cognitive Failure, Anxiety, Latent Moderated Structural Equation

\section{(c) (i) Open Access}

\section{Introduction}

According to the data of Ministry of Education (2019), the number of college students in our country reached to 28.31 million people in 2019, and the undergraduate students are about 16.97 million. Studies have showed that here is a phenomenon of learning burnout among local university students ( $\mathrm{Li} \&$ Cheng, 2016; Huang \& Yu, 2010). Among them, the rate of severe burnout is about $44.72 \%$, moderate burnout $50.93 \%$ and mild burnout $4.35 \%$. It mainly involves feeling exhausted, unable to maintain a long-term enthusiasm for learning, sleeping in class, playing mobile phones and other bad habits (Song \& Xie, 2019).

College students mainly use mobile phones in the process of walking, classing and eating, which will bring adverse impact on their mental and physical health and will also bring many problems to our society (Zhang, Xiao, \& Zhu, 2019). It 
can be seen that learning burnout and mobile phone addiction are widespread in college students' daily life, and have a negative impact on individuals and society. Therefore, it will be helpful to improve this situation by researching the relationship between the phone addiction and learning burnout.

\subsection{The Relationship between the Phone Addiction and Learning Burnout}

Regarding to the phone addiction, some researchers point out that it is a kind of addictive behavior, also known as mobile phone dependence, caused by excessive addiction to various activities of mobile phones, which leads to a strong and continuous sense of craving and dependence, and leads to obvious impaired social and psychological functions (Cheng et al., 2008). Others scholars believe that the physical and psychological discomfort symptoms is caused by improper use of mobile phones (Xiong, Zhou, Chen, You, \& Zhai et al., 2012). They also think that mobile phone addiction is a compensatory behavior (Rozgonjuk, Levine, \& Hall et al., 2018). But the present research points out that (Brand, Young, \& Laier, 2016): Mobile phone addiction is the result of the interaction of personalityemotion-cognition-execution in the environment. After these four factors, the mobile phone addiction produces satisfaction, which leads to the compensation effect. Some studies have sorted out previous achievements (Zhang, Cheng et al., 2019), believing that mobile phone addiction will harm individuals' psychological and physical health, mainly damaging human organs, sleep and physical quality, resulting in mental illness, cognitive failure and subjective perception. Through individual behavior, mobile phone addiction will affect the individual's work efficiency, academic performance, future planning and interpersonal relationship. At the same time, individual behavior will further to affect the parent-child relationship, traffic safety and even violate the law and morality.

As for learning burnout, some scholars think that it is a phenomenon that students' energy is exhausted due to long-term academic pressure, their enthusiasm for learning and activities is gradually weakened, their attitude towards classmates is alienated and have a negative attitude towards learning (Meier \& Schmeck, 1985). Others researchers believe that it is the behavior that students have no interest in learning or lack motivation but have to avoid learning (Lian, Yang, \& Wu, 2005). Studies have found that different grades in college students have significant differences in learning burnout (Song \& Xie, 2019; Li et al., 2012). With the higher grade of college students, the more serious learning burnout they will have. In addition to this, they found that the boys' learning burnout is higher than girls'. The main factor which causes learning burnout is the individual factor (Song \& Xie, 2019), like that motivation, sense of accomplishment, methods, etc. Secondly, school management and atmosphere, employment pressure may also produce learning burnout. Some researches also found that it can positively predict sleep quality through learning burnout, which indicates that learning burnout can affect college students' sleep quality (Zhang, 
Yang, Zhang, Ning et al., 2016).

There is a significant correlation between mobile phone addiction and learning burnout (Zhang \& Shen, 2015; Li, \&Jin, 2018), students with high degree of mobile phone addiction are more likely to have learning burnout (Ge, 2013). Some studies have showed that mobile phone addiction can affect learning efficiency and generate learning burnout (Zhang, Cheng, Zhang, \& Xiao, 2019; Qu, Lu, Song et al., 2017). When individuals encounter difficult-to-adjust emotions, they will have the sense of self-inconsistency, and it is easy to use new skills or resources to achieve balance. The information provided by mobile phones is the one of the most accessible for college students. The most significant feature of college students' excessive use of mobile phones is that they spend too much time on mobile phones, even sacrificing sleep and class time (Li \& Yuan, 2016). However, learning is a process of continuous accumulation. College students put too much energy into the information provided by mobile phones, which makes it difficult for them to shift their attention to study in time and ultimately fail to carry out their study smoothly (Song \& Xie, 2019; Zhang, Cheng et al., 2019). While some of them are addicted to mobile games or network virtual interpersonal relationships, and are often involuntarily divorced from the real society (Qu et al., 2017). In the same way, they are unwilling to participate in the normal classroom discussion, which not only leads to learning burnout, but also seriously affects the normal life. In addition, college students who are addicted to mobile phones are unable to isolate their phones quickly, and they will check their phones from time to time (even if the mobile phone don't provide any information), which also results in college students' inability to fully devote themselves to learning, thus further aggravates their learning burnout (Xiong et al., 2012). Research found that the higher degree of mobile phones addiction will take up more time of learning, which will affect normal rest and increase the sense of fatigue (Zhang \& Shen, 2015). At the same time, it will ignore the self-supervision in learning which will lead to the declining of learning efficiency, and then result in weariness and low sense of achievement (Wang, Yu, \& Yang, 2007).

\subsection{The Function of Cognitive Failure}

Cognitive failure is the phenomenon that an individual makes mistake due to cognitive factors when completing simple tasks in daily life (Zhang \& Zhang, 2011). The research concluded that (Zhou et al., 2016): Cognitive failures are not only related to cognition and personality, but also causes many mental health problems and social problems. Among them, psychologists pay more attention to the general tendency of mistakes in typical behaviors and habitual behaviors, which mainly involves the allocation of cognitive resources in multitasking and the key is the transfer of attention (Klumb, 2001). One of the manifestations of cognitive failure is absent-mindedness. Absent-minded people have difficulty paying attention to activities for a long time, so their attention is easily attracted by the current irrelevant stimuli, thereby neglecting the ongoing activities and 
losing track of the current target. The ability of keeping attention is likely to play an important role in the individual's tendency towards cognitive failure (Manly, Robertson, Galloway et al., 1999). Overuse mobile phone will lead to a sense of fatigue, distracted and easy to be absent-minded. Prolonged concentration on the stimulation provided by the phone may also lead to an inability to migrate to the current activity. The research also found that the mobile phones addiction can positively predict cognitive failure (He \& Xia, 2019). When individual spend too much energy on mobile phone, excessive cognitive resources consumption leads to students' inability to fully devote themselves to learning, which is prone to energy loss and mental exhaustion, and resulting in learning burnout ( $\mathrm{Wu}$, Dai, Wen, \& Cui, 2010).

\subsection{Research Hypothesis}

As a negative emotion, anxiety not only affects mental health, but also affects emotional eating and harms people's health (Liu et al., 2016). Some studies suggest that negative emotions can promote the behavior suppression in college students who are trait and anxious (Jia et al., 2018). Studies have found that there is a significant positive correlation relationship between anxiety and mobile phone addiction (Gao, Zhang et al., 2019; Hu, Huang et al., 2017). When college students are faced with inescapable pressure (such as academic pressure), they will have negative emotions, which may drive individuals to take negative coping methods, such as indulging in "safe" network information provided by mobile phones. At the same time, mobile phone addiction will produce more negative emotions, Individuals with high levels of phone addiction had higher levels of anxiety (Mecacci, Righi, \& Rocchetti, 2004). Individuals with high negative emotions may have low self-esteem and negative self-cognition, which leads to the result that they're unable to adopt effective cognitive strategies when faced with stressful situations. In addition, it is difficult for them to complete cognitive tasks successfully, which will result in more cognitive failures. But research points out that (Peng, 2015): The relationship between anxiety and work efficiency is not linear, and the moderate tension and anxiety can promote individuals to think and solve problems actively. The relationship between the two shows an inverted u-shaped curve, and too high or too low degree of emotional arousal is not conducive to the individual to complete the current task. Overuse of mobile phones will make the undergraduates reduce their learning efficiency. It is inevitable for students to have the emotion of anxiety when they are faced with the problems like final exams and employment selection. However, in the case of cognitive failure, this anxiety will promote the individuals to adopt a positive method or take a laissez-faire attitude? At present, only a few studies have investigated the relationship among anxiety, cognitive failure, learning burnout and mobile phone addiction tendency, so this research assumes that the mobile phone addiction can positively predict learning burnout, cognitive failure plays an mediating role, and anxiety as a negative emotion plays a moderating role. 


\section{Research Methods}

\subsection{Participants}

Participants were randomly selected from Guizhou province in China. 600 questionnaires were received and the valid sample included 596 college students $($ male $=135$; female $=461)$. Participants' age ranged from 17 to 25 years old with a mean age of $20.25(S D=1.16)$. Among them, freshman $=5$, sophomore $=451$, junior $=110$, senior $=30$. And there were 469 people from the countryside and 127 from the cities.

\subsection{Analytical Approach}

In this study, SPSS25.0 was used to conduct descriptive analysis, correlation analysis. Mplus8.3 was used for common method variance analysis, confirmatory factor analysis, and bias-corrected Bootstrap method was used to analysis the mediating effect and moderated effect. Firstly, we conducted a preliminary analysis which consists of four parts: 1) Confirmatory factor analysis was utilized to confirm the construct validity; 2) Verified that reliability and validity were up to standards; 3) Descriptive statistics and bivariate correlations were presented; 4) Common method variance analysis were used to rule out method variance. Secondly, the mediating effect of cognitive failure was conducted. More importantly, latent moderated structural equation was adopted to test the moderated effect. Finally, the interactions were depicted using the high (1 SD above the mean) and low (1 SD below the mean) scores to analyze the moderated effect of anxiety.

\subsection{Research Tools}

\subsubsection{Anxiety Scale (AX)}

The Anxiety Sub Scale of the Symptom Checklist 90 Scale (SCL-90) was developed by Derogatis (Wang, Wang, \& Ma, 1999). The scale has 10 items, which are all rated on 5-point Likert scale $(0=$ never, $4=$ almost always $)$.When the total score of this scale is high, the individuals had more anxiety experience. In this study, the Cronbach $\alpha$ coefficient of the scale is 0.95 , the average variance extracted (AVE) value is 0.65 . The fit index of confirmatory factor analysis is good $\left(\chi^{2} / \mathrm{df}=4.18 ; \mathrm{CFI}=0.96 ; \mathrm{TLI}=0.95 ; \mathrm{SRMR}=0.03 ; \mathrm{RMSEA}=0.07\right)$.

\subsubsection{The College Students' Cognitive Failure Scale (CF)}

The College Students' Cognitive Failure Scale was developed by Zhang Lin (Zhang, 2013). The scale has 18 items for measuring individual level of Cognitive Failure, all the items are all rated on 5 -point Likert scale $(1=$ very disagree, $5=$ very agree). This scale includes three dimensions as attention failure, memory failure, and action function failure, and it is used to measure the individual's Cognitive Failure experience. In this study, the Cronbach $\alpha$ coefficient of the scale is 0.95 , the average variance extracted (AVE) value is 0.50 . The fit index of confirmatory factor analysis is good $\left(\chi^{2} / \mathrm{df}=3.41\right.$; CFI $=0.93$; TLI $=0.92$; SRMR $=0.05$; RMSEA $=0.06$ ). 


\subsubsection{The College Student Learning Burnout Scale (LB)}

The College Student Learning Burnout Scale was developed to measure the individuals' learning status by Lian Rong et al. (Lian, Yang, \& Wu, 2005). The scale has 20 items for measuring individual level of Learning Burnout, all the items are all rated on 5 -point Likert scale ( $1=$ very disagree, $5=$ very agree). This scale includes three dimensions of improper behavior, depression, and low sense of achievement. When the total score of this scale is high, the individuals had more Learning Burnout experience. In this study, the Cronbach a coefficient of the scale is 0.88 , the average variance extracted (AVE) value is 0.31 . The fit index of confirmatory factor analysis is good $\left(\chi^{2} / \mathrm{df}=2.47\right.$; CFI $=0.93$; TLI $=0.92$; $\mathrm{SRMR}$ $=0.05 ;$ RMSEA $=0.05$ ).

\subsubsection{The University Student Mobile Phone Addiction Tendency Scale (MPATS)}

The University Student Mobile Phone Addiction Tendency Scale was developed by Xiong Jie et al. (Xiong et al., 2012), which is designed to assess one's the degree of mobile phone addiction. The MPATS has 16 items, which are all rated on 5 -point Likert scale ( $1=$ very disagree, $5=$ very agree). This scale includes four dimensions of withdrawal symptoms, prominent behavior, social comfort, and mood changes. When the total score of this scale is high, the individuals had more phone addiction tendency. In this study, the Cronbach a coefficient of the scale is 0.94 , the average variance extracted (AVE) value is 0.48 . The fit index of confirmatory factor analysis is good $\left(\chi^{2} / \mathrm{df}=3.91\right.$; CFI $=0.92$; TLI $=0.90$; $\mathrm{SRMR}$ $=0.05$; RMSEA $=0.07$ ).

\section{Research Result}

\subsection{Descriptive Statistics}

In this study, there are many complex models and a large number of estimated parameters, so we use Item Parceling Method to simplify the model. According to the scholars' suggestion (Wu \& Wen, 2011), the measurement model has been analyzed on each scale before using Item Parceling Method. For the anxiety scale, according to the load value of each item factor, the High-High Loading Method is adopted to parcel the items into two groups. So as to reduce the difference in groups and increase the index consistency, the Internal Consistency Method is used in the scale of Learning Burnout, Cognitive Failure and Mobile Phone Addiction Tendency. The items under the same dimensions are parceled as an indicator to emphasize the consistency of the purpose of the questions on each group.

The results show that (see Table 1 ), all variables are positively correlated (all $p$ $<0.01$ ). The Skewness coefficient (S) after parceling is between -0.42 and 0.27 , the kurtosis coefficient $(\mathrm{K})$ is between -0.34 and 1.41, and it approximates normal distribution. According to the suggestion (Wang, 2005), it is robust to use the ML estimation when the skewness coefficient is less than 2 and the kurtosis coefficient is less than 7 . 


\section{Common Method Variance Analysis}

Common method deviation analysis is performed on the parceled data. According to the researchers' suggestions (Wen, Huang, \& Tang, 2018), after adding the method factor, if CFI and TLI increase by more than 0.1 and RMSEA and SRMR decrease by more than 0.05 , it indicates that there is a serious common method variance. The results show that CFI and TLI increased by 0.01 , and SRMR and RMSEA are almost unchanged (see Table 2). It also shows that the model not improves significantly after adding the method factor. It demonstrates that the common method variance is not obvious.

\subsection{Structural Model}

\subsubsection{Mediating Model Analysis}

Based on the theoretical hypothesis, the structural equation model is established, and the fitting indexes reach the standard $\left(\chi^{2} / \mathrm{df}=4.80\right.$; $\mathrm{CFI}=0.96$; $\mathrm{TLI}=0.94$; SRMR $=0.04$; RMSEA $=0.08$ ).

The results show that (see Table 3), the prediction effect of mobile phone addiction on learning burnout is significant (effect $=0.51, Z=10.75, p<0.001$ ). After joining the cognitive failure, the direct predictive effect on mobile phone addiction to learning burnout is significant (effect $=0.17, Z=3.01, p<0.01$ ). The positive predictive effect of mobile phone addiction on cognitive failure is significant (effect $=0.57, Z=12.32, p<0.001$ ), cognitive failure has a significant positive predictive effect on learning burnout (effect $=0.59, Z=8.70, p<0.001$ ). It indicates that the mobile phone addiction can not only directly predict learning burnout, but also can predict learning burnout through the mediating role of cognitive failure. Among them, the direct effect (0.17) and the mediating effect $(0.34)$ accounted for $33.33 \%$ and $66.67 \%$ of the total effect $(0.51)$, respectively (see Table 4).

Table 1. Descriptive analysis and correlation matrix of each variable in the study $(\mathrm{n}=$ 596).

\begin{tabular}{cccccc}
\hline Index & $x \pm s$ & 1 & 2 & 3 & 4 \\
\hline 1) $A X$ & $14.02 \pm 0.33$ & 1 & & & \\
2) $C F$ & $51.75 \pm 0.40$ & $0.60^{* *}$ & 1 & & \\
3) $L B$ & $55.18 \pm 0.45$ & $0.51^{* *}$ & $0.61^{* *}$ & 1 & \\
4) $M P A T S$ & $45.82 \pm 0.45$ & $0.51^{* *}$ & $0.60^{* *}$ & $0.47^{* *}$ & 1 \\
\hline
\end{tabular}

Table 2. Common method variance analysis.

\begin{tabular}{|c|c|c|c|c|c|c|c|c|}
\hline$M L$ & $\chi^{2}$ & $d f$ & $C F I$ & $T L I$ & $A I C$ & $B I C$ & $S R M R$ & $\begin{array}{l}\text { RMSEA } \\
(90 \% C I)\end{array}$ \\
\hline $\begin{array}{c}\text { Measurement } \\
\text { model }\end{array}$ & 265.38 & 38 & 0.96 & 0.95 & $10,118.37$ & $10,302.76$ & 0.04 & $\begin{array}{c}0.08 \\
{[0.08,0.10]}\end{array}$ \\
\hline $\begin{array}{l}\text { Bifactor } \\
\text { model }\end{array}$ & 189.45 & 34 & 0.97 & 0.96 & $10,054.44$ & $10,265.17$ & 0.04 & $\begin{array}{c}0.08 \\
{[0.06,0.09]}\end{array}$ \\
\hline$\Delta$ & 75.93 & 4 & -0.01 & -0.01 & -63.93 & -37.59 & 0.00 & 0.00 \\
\hline
\end{tabular}


Table 3. Mediating effect analysis of cognitive failure.

\begin{tabular}{ccccccc}
\hline$D V$ & $I V$ & Estimate & S.E. & Est./S.E. & Lower 2.5\% & Upper $2.5 \%$ \\
\hline LB & MPATS & 0.51 & 0.05 & $10.75^{* * *}$ & 0.40 & 0.58 \\
$C F$ & $M P A T S$ & 0.57 & 0.05 & $12.32^{* * *}$ & 0.47 & 0.66 \\
LB & $M P A T S$ & 0.17 & 0.06 & $3.01^{* *}$ & 0.05 & 0.27 \\
& $C F$ & 0.59 & 0.07 & $8.70^{* * *}$ & 0.46 & 0.73 \\
\hline
\end{tabular}

Note: DV: the outcome variable, IV: the predictor variable; ${ }^{*}: p<0.05,{ }^{* *}: p<0.01,{ }^{* * *}: p<0.001$; Lower $2.5 \%$ and Upper $2.5 \%$ refer to the interval estimates of BCBootstrap $2.5 \%$ and $97.5 \%$. Same as below.

Table 4. Total effect, direct effect and the mediating effect of breakdown.

\begin{tabular}{ccccccc}
\hline & Estimate & S.E. & Est./S.E. & Lower 2.5\% & Upper 2.5\% & Percentage \\
\hline Direct effect & 0.17 & 0.06 & $3.01^{* *}$ & 0.05 & 0.27 & $33.33 \%$ \\
Indirect effect & 0.34 & 0.05 & $7.34^{* * *}$ & 0.25 & 0.43 & $66.67 \%$ \\
Total effect & 0.51 & 0.05 & $10.75^{* *}$ & 0.40 & 0.58 & \\
\hline
\end{tabular}

\subsubsection{The Moderated Mediation Model Fitting Analysis}

According to the suggestions of researcher (Fang \& Wen, 2018) and took the MPLUS program based on the LMS method. The first step is to analyze the benchmark model without latent moderated term. The benchmark model is fitted like below: $\chi^{2} / \mathrm{df}=4.65, \mathrm{CFI}=0.95, \mathrm{TLI}=0.94, \mathrm{AIC}=10183.66, \mathrm{BIC}=$ $10,316.00$, SRMR $=0.04$, RMSEA $=0.08$ (90\% CI: $[0.07,0.09])$. It indicates that benchmark SEM model is reasonable.

The second step is to analyze the SEM model with latent moderated term. Since Mplus does not provide common fitting indexes, such as CFI, TLI, SRMR, RMSEA, etc., when executing LMS, and it can be achieved by AIC and likelihood comparison for the fitting indexes (Fang \& Wen, 2018). 1) AIC analysis. The benchmark model AIC $=10,183.66$, the latent moderated item SEM model AIC $=10,136.00$, the AIC decreased by 47.66, it shows that the fitted index of the moderated mediation model has improved compared with the benchmark model; 2) Likelihood ratio analysis. Log Likelihood of the moderated mediation model $=-5027.00$, Log Likelihood of the benchmark model $=-5048.83$, increased by 21.83 , we also can see that, -2 LL value is 43.66 , the degree of freedom increases by 2 , the chi-square analysis of -2 LL value is significant ( $\mathrm{p}<$ 0.01 ), it indicates that the moderated mediation model is better than the benchmark model. Both of these two analysis results demonstrate that the moderated mediation model is acceptable.

\subsubsection{The Moderated Mediation Effect Analysis}

When put the anxiety into the model, the results show that the anxiety and mobile phone addiction has a significant predicting effect to the cognitive failure (effect $=0.11, \mathrm{Z}=2.32, p<0.05$ ) (see Table 5). We use Product Coefficient Method, the confidence interval of $a_{3} b_{1}$ is $[0.01,0.08]$, and 0 is not included in the interval. This indicates that the product item $\mathrm{a}_{3} \mathrm{~b}_{1}$ is significant and we also can get the conclusion that the mediating effect of mobile phone addiction on learning burnout through cognitive failure is moderated by anxiety, and only mod- 
erated the first half of the mediating pathway.

Further analysis shows that the moderated effect is enhanced effect (see Table $6)$, the mediating effect is significant when the anxiety was -0.57 (M - 1 SD) (effect $=0.07, Z=3.23, p<0.01$ ); the mediating effect is significant when the anxiety was $0.54(\mathrm{M}+1 \mathrm{SD})($ effect $=0.11, \mathrm{Z}=3.79, p<0.001)$. (Among them, the limits of the $95 \%$ confidence interval do not include 0 ).

\section{Discussion}

\subsection{The Mediating Effect of Cognitive Failure}

The research results show that college students' mobile phone addiction has a positive predictive effect on learning burnout, it is in consistent with studies in past years (Zhang, Cheng et al., 2019; Qu et al., 2017). Immersion Theory points out that mobile phone addicts will pay full attention to the information provided by mobile phone, and their self-awareness and sense of time will disappear (Csikszentmihaly, 1990). This pleasant experience will lead to maintain this state at a higher cost. Mobile phone addicts voluntarily reduce the learning time and sleeping time, resulting in a significant decline in learning efficiency (Liu et al., 2016). This indicates that college students are addicted to mobile phones, which leads to a decrease in learning time and reduces effective participation in class discussions. In addition, with the open teaching in universities, the time for college students to control themselves increases. College students overuse mobile phones, once they become dependent on them, they will spend a lot of energy and attention in the Internet world, which will cause serious withdrawal symptoms. It also damages the physiological and psychological functions, such as reducing the cognitive resources of attention, reducing the capacity of working memory, and reducing the time of continuous attention (Jonathan, Carriere, Allan, \& Daniel, 2007). The pleasure and virtual interpersonal interactions brought by the mobile phones are not available in the classroom. In addition, the flexibility of university classroom content is relatively weak, which is mainly based on the theoretical basic knowledge. Therefore, students are prone to develop a weariness of learning and "people are absent" situation will also occurs, which finally leads to learning burnout.

Table 5. Moderated mediation effect analysis.

\begin{tabular}{ccccccc}
\hline DV & $I V$ & Estimate & S.E. & Est./S.E. & Lower 2.5\% & Upper $2.5 \%$ \\
\hline \multirow{4}{*}{$C F$} & MPATS & 0.26 & 0.06 & $4.03^{* * *}$ & 0.12 & 0.37 \\
& $A X$ & 0.68 & 0.07 & $9.26^{* * *}$ & 0.56 & 0.84 \\
& $I N T\left(\right.$ MPATS $\left.^{*} A X\right)$ & 0.11 & 0.05 & $2.32^{*}$ & 0.01 & 0.20 \\
\hline
\end{tabular}

Table 6. Simple effect analysis.

\begin{tabular}{ccccccc}
\hline & $A X$ & Estimate & S.E. & Est./S.E. & Lower $2.5 \%$ & Upper $2.5 \%$ \\
\hline \multirow{2}{*}{ Mediation } & $-0.57(M-1 S D)$ & 0.07 & 0.02 & $3.23^{* *}$ & 0.03 & 0.11 \\
& $0.54(M+1 S D)$ & 0.11 & 0.03 & $3.79^{* * *}$ & 0.06 & 0.18 \\
\hline
\end{tabular}


The cognitive failure plays a mediating effect between mobile phone addiction and learning burnout, this shows that the mobile phone addiction can also affect learning burnout through cognitive failure. When undergraduates use the mobile phones for a long time and pay much attention to it, they will appear absent-minded and can't really devote to learning. However, sometimes the university knowledge is coherent. If students are lack of a certain link, it will obviously cause weakness in subsequent learning, Which will easily lead to physical and mental exhaustion and inability to concentrate. At the same time, excessive attention to the information provided by the mobile phone will affect the transfer of attention (Ball-Rokeach \& De-Fleur, 1976). Mobile phone addicts cannot timely shift their attention to the current learning activities without enough practice or knowledge transfer. When they engage in an activity that requires the application of knowledge, they will fail in action functions. Such long-term lack of learning gradually leads to weariness and learning burnout.

\subsection{Moderated Effect of Anxiety}

The results show that the anxiety can moderate the mediating role of cognitive failure, and moderating the first half of the mediating effect (see Figure 1), we also can say that the anxiety moderates a role of mobile phone addiction on positively predicting cognitive failure. According to Media Dependence Theory, the more individuals rely on media to meet their needs, the greater the proportion of media in their lives will be, and the stronger the media's influence on individuals will be (Zuckerman, Eysenck, \& Eysenck, 1978). Since it is impossible for people to live in a "vacuum" state, so certain stimulus input is necessary (Ding, Cui, \& Wang, 1995). The most convenient way for college students to seek stimulation is mobile phones. However, the overuse of mobile phones leads to excessive consumption of cognitive resources. When faced with other tasks, it is difficult for them to transfer their attention to support the normal continuation of other tasks. Self-Inconsistent Theory thinks that people's general anxiety is caused by the inconsistency between the actual self and the hoped self (Chen \& Wang, 2009). Even when college students are addicted to mobile phones, the hoped self and the actual self are likely to be at odds, and this cognitive bias leads to widespread anxiety. Under the premise of excessive consumption of cognitive resources, anxiety will magnify this inconsistency and continue to consume cognitive resources, which makes it more difficult for individuals to concentrate and

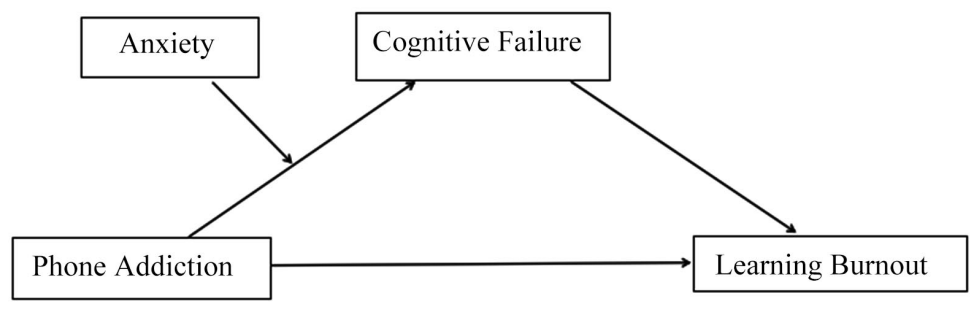

Figure 1. The influence of phone addiction on learning burnout. 
transfer their attention. The study found that moderate anxiety can help individuals to actively cope with the current tasks (Zhang, Li, \& Yu, 2019). But the study ignores that under the premise of excessive consumption of cognitive resources, individuals can no longer block environmental interference. When the cognitive resources are exhausted and the attention is hard to concentrate, it is difficult for individuals to go on their learning, and even memory failure and action function failure will occur (Xiong et al., 2012).

\section{Implications}

The study found that college students' excessive use of mobile phones will lead to learning burnout, indicating that cell phone addiction has affected the normal learning of college students. At the same time, phone addiction can affect cognitive failure, which in turn affects burnout behavior. After the excessive consumption of cognitive resources, anxiety will aggravate learning burnout, which will also result in behavioral and psychological problems. The research results conclude that mobile phone addiction and cognitive failure can cause learning burnout. Therefore, we should guide college students to use mobile phones rationally and train their ability to cope with anxiety. More importantly, we should encourage college students to actively participate in class discussions, constantly learn new knowledge and skills, and avoid falling into a vicious cycle of learning burnout.

\section{Limitations and Suggestions for Future Research}

There are still some limitations in this research, it can be improved in the future. Firstly, we only invited some students in Guizhou Normal University to take part in this research, so the sample is underrepresented; secondly, the anxiety is too broad, it can distinguish them in future research, such as test anxiety, interpersonal anxiety and so on and find out the specific effects of anxiety; Thirdly, whether the moderated effect of anxiety is also significant in other pathways remains to be further studies.

\section{Conclusion}

1) The phone addiction tendency has a positively significant effect on learning burnout;

2) The phone addiction tendency can influence learning burnout through cognitive failure;

3) Anxiety can moderate the mediating role of cognitive failure.

\section{Funding}

Guiyang Baiyun District Science and Technology Bureau project.

\section{Conflicts of Interest}

The authors declare no conflicts of interest regarding the publication of this paper. 


\section{References}

Ball-Rokeach, S. J., \& De-Fleur, M. L. (1976). A Dependency Model of Mass-Media Effects. Communication Research, 3, 3-21. https://doi.org/10.1177/009365027600300101

Brand, M., Young, K. S., \& Laier, C. (2016). Integrating Psychological and Neurobiological Considerations Regarding the Development and Maintenance of Specific Internet-Use Disorders: An Interaction of Person-Affect-Cognition-Execution (I-PACE) Model. Neuroscience \& Biobehavioral Reviews, 71, 252-266. https://doi.org/10.1016/j.neubiorev.2016.08.033

Chen, J., \& Wang, D. Y. (2009). Review on Study of Existential Anxiety. Progress in Psychological Science, 17, 204-209.

Cheng, F. Y., Tang, T. C., Yen, J. Y., Lin, H. C., Huang, C. F., Liu, S. C., Ko, C. H. et al. (2008). Symptoms of Problematic Cellular Phone Use, Functional Impairment and Association with Depression among Adolescents in Southern Taiwan. Journal of Adolescence, 32, 863-873. https://doi.org/10.1016/j.adolescence.2008.10.006

Csikszentmihaly, M. (1990). Flow: The Psychology of Optimal Experience. New York: Harper Perennial Modern Classics.

Ding, J. H., Cui, W., \& Wang, H. H. (1995). A Preliminary Study on Anxiety of College Students. Chinese Journal of Mental Health, No. 4, $153+152$.

Fang, J., \& Wen, Z. L. (2018). Analysis of Moderated Mediating Effects Based on Structural Equation Model. Psychological Science, 41, 453-458.

Gao, D. P., Zhang, X. X., Sun, Y. C., \& Jia, L. P. (2019). The Relationship between Cell Phone Addiction and Loneliness and Anxiety of Medical College Students in Weifang. Medicine and Society, 32, 95-98.

Ge, S. H. (2013). The Relationship between Mobile Addiction and Learning Burnout of Secondary Vocational School Students. Mental Health Education in Primary and Secondary School, No. 15, 14-15 + 17.

He, A. M., \& Xia, Y. Y. (2019). The Influence of Cell Phone Addiction on College Students' Cognitive Failure: A Moderated Mediation Model. Psychological Development and Education, 35, 295-302.

Hu, Y., Huang, H., Zhang, Y. Q., \& Zhou, C. Y. (2017). Relationship between Mobile Phone Dependence and Cognitive Failure in College Students: Mediating Effect of Negative Emotions. Chinese Journal of Clinical Psychology, 25, 1088-1092.

Huang, S. H., \&Yu, D. (2010). A Survey of Mobile Phone Use and Dependence of College Students in Guangzhou. Health Soft Science, 24, 252-254 + 261.

Jia, L. P., Bai, X. J., Shao, J. G., Lu, G. H., Han, X. X., \& Li, C. J. (2018). The Influence of Emotion Induction on Behavior Inhibition of Students with Trait Anxiety. China Journal of Mental Health, 32, 1029-1033.

Jonathan, S. A., Carriere, J., Allan, C., \& Daniel, S. (2007). Everyday Attention Lapses and Memory Failures: The Affective Consequences of Mindlessness. Consciousness and Cognition, 17, 835-847. https://doi.org/10.1016/j.concog.2007.04.008

Klumb, P. L. (2001). Tying Knots in Handkerchiefs: The Use of Memor Aids in Everyday Life. Zeitschrift fur Entwicklungs Psychologie and Padagogische Psychology, 33, 42-49. https://doi.org/10.1026//0049-8637.33.1.42

Li, D. B., \& Yuan, C. (2016). The Current Situation and Causes of College Students' Learning Burnout. China Health Industry, 13, 30-33.

Li, F. Y., Zhang, P., Liu, J. W., Zhang, W. W., Ding, Y., Chen, Y. W., Yin, C. J., Tuo, X. Q., Li, Y. Y. et al. (2012). Analysis of Learning Burnout and Influencing Factors of Xinjiang 
University Students. China Public Health, 28, 1104-1105.

Li, S. J., \& Jin, C. C. (2018). The Relationship between Mobile Phone Dependence and Learning Burnout of College Students: The Moderated Role of Personality. China Special Education, No. 5, 86-91.

Lian, R., Yang, L. X., Wu, L. H. et al. (2005). The Relationship between Professional Commitment and Learning Burnout of College Students and the Development of Scale. Journal of Psychology, No. 5, 632-636.

Liu, W. L., Cai, T. S., Zhu, H., Lu, Y., \& Ling, Y. (2016). The Relationship between Depression, Anxiety, Stress and Adolescents' Emotional Eating: Mediating Role of SelfControl. Chinese Journal of Clinical Psychology, 24, 841-843.

Manly, T., Robertson, I. H., Galloway, M. et al. (1999). The Absent Mind: Further Investigation of Sustained Attention to Response. Neuropsychologia, 37, 661-670. https://doi.org/10.1016/S0028-3932(98)00127-4

Mecacci, L., Righi, S., \& Rocchetti, G. (2004). Cognitive Failures and Circadian Typology. Personality \&Individual Differences, 37, 107-113. https://doi.org/10.1016/j.paid.2003.08.004

Meier, S. F., \& Schmeck, R. (1985). The Burned Out College Student: A Descriptive Profile. Journal of College Student Personal, 26, 63-69.

Peng, D. L. (2015). General Psychology (p. 409). Beijing: Beijing Normal University Press.

Qu, X. Y., Lu, A. T., Song, P. F., Lan, Y. L., Cai, R. Y. et al. (2017). The Influence of Mobile Phone Addiction on Learning Burnout: Mediated by Academic Procrastination. Applied Psychology, 23, 49-57.

Rozgonjuk, D., Levine, J. C., Hall, B. J. et al. (2018). The Association between Problematic Smart Phone Use, Depression and Anxiety Symptom Severity, and Objectively Measured Smart Phone Overuse One Week. Computers in Human Behavior, 87, 10-17. https://doi.org/10.1016/j.chb.2018.05.019

Song, N. Q., \& Xie, X. J. (2019). Study on the Current Situation and Countermeasures of Undergraduates' Learning Burnout-Taking Southwest China as an Example. Teaching of China University, $z 1,93-97+101$.

Wang, B., Yu, H. B., \& Yang, S. (2007). The Relationship between Online Game Addiction and Learning Burnout of College Students. Chinese Journal of Mental Health, No. 12, 841-844.

Wang, X. D., Wang, X. L., \& Ma, H. (1999). Handbook of China Mental Health Assessment Scale (Updated Version) (pp. 31-35 + 83-86). Beijing: China Mental Health Journal Press.

Wang, X. M. (2005). Applied Probability Statistics. Shanghai: Shanghai University of Finance and Economics Press.

Wen, Z. L., Huang, B. B., \& Tang, D. D. (2018). Prequel to Questionnaire Data Modeling. Psychological Science, 41, 204-210. https://doi.org/10.1016/j.apsusc.2017.10.187

Wu, Y., \& Wen, Z. L. (2011). Item Parceling Strategy in Structural Equation Modeling. Progress in Psychological Science, 19, 1859-1867.

Wu, Y., Dai, X. Y., Wen, Z. L., \& Cui, H. Q. (2010). Development of Adolescent Learning Burnout Scale. Chinese Journal of Clinical Psychology, 18, 152-154.

Xiong, J., Zhou, Z. K., Chen, W., You, Z. Q., Zhai, Z. Y. et al. (2012). Development of Mobile Phone Addiction Tendency Scale for College Students. China Journal of Mental Health, 26, 222-225. https://doi.org/10.1037/t74211-000

Zhang, B., Cheng, S. Z., Zhang, Y. J., Xiao, W. et al. (2019). Analysis of Mobile Phone Addiction and Academic Burnout among Medical Students: Mediating Role of Self- 
Control. Chinese Journal of Health Psychology, 27, 435-438.

Zhang, H. M., \& Zhang, Z. J. (2011). A Review of Cognitive Failure: Concepts, Measurement and Related Research. Psychological Innovation, 31, 89-93.

Zhang, L. (2013). Development of Cognitive Failure Questionnaire for College Students. Chongqing: Southwest University.

Zhang, M., Xiao, Q., \& Zhu, L. G. (2019). Research Progress on Antecedents, Outcomes and Interventions of Mobile Phone Dependence. China Special Education, No. 11, 88-96.

Zhang, S. X., Yang, X. Y., Zhang, Y., Ning, L. et al. (2016). Correlation Analysis between Sleep Quality and Learning Burnout of Medical College Students in Xinjiang. China Occupational Medicine, 43, 181-184.

Zhang, X. W., \& Shen, Q. Y. (2015). A Study on the Relationship between Mobile Phone Dependence and Learning Burnout of College Students Based on an Empirical Analysis of Vocational College Students. Career Time and Space, 11, 96-99.

Zhang, Y. L., Li, S., \& Yu, G. L. (2019). The Relationship between Boredom Tendency and Cognitive Failure of College Students: The Mediating Effect of Mobile Phone Addiction Tendency and Difference between the Only Child and Non Only Child Groups. Psychological Development and Education, 35, 344-351.

Zhou, Y., Chen, J. Z., Liu, Y., Wang, P., Zhu, L. J., \& Yan, T. H. (2016). The Validity and Reliability of the Chinese Version of the Cognitive Failure Questionnaire for College Students. Chinese Journal of Clinical Psychology, 24, 438-443.

Zuckerman, M., Eysenck, S., \& Eysenck, H. J. (1978). Sensation Seeking in England and America: Cross-Cultural, Age, and Sex Comparisons. Journal of Consulting and Clinical Psychology, 46, 139-149. https://doi.org/10.1037/0022-006X.46.1.139 